\title{
A local view on single and coupled moleculesq.
}

\author{
Niek van Hulst** \\ Erik van Dijk, Jordi Hernando ${ }^{+}$, Jacob Hoogenboom, Mar a Garc a-Paraj \\ Applied Optics group, Faculty of Science \& Technology, \\ $\mathrm{MESA}^{+}$Institute for Nanotechnology, University of Twente, the Netherlands. \\ ${ }^{+}$Current address Universidad Autonoma Barcelona, Spain \\ *N.F.vanHulst@utwente.nlwwww.tn.utwente.nl/ot
}

Rapid advances in NanoPhotonics now allow the exploration and manipulation of light in and around nanostructures, single molecules, molecular complexes, etc. Indeed by proper control on the nm-scale subwavelength light fields are being created and detected, where large (imaginary) wave vectors dominate, resulting in strong field confinement, sharp field gradients, photon tunneling, (plasmonic) resonances, extraordinary transmission, etc.

Surprisingly enough ultrafast fs-ps laser-spectroscopy, one of the major advantages of photonics, has hardly been exploited at the nanometer scale, let alone on single molecules or nanoparticles. Indeed femtosecond nanophotonics is experimentally challenging, but once mastered full of novel promises. Here we will focus on a novel approach to reveal ultrafast dynamics in single molecules (figure).

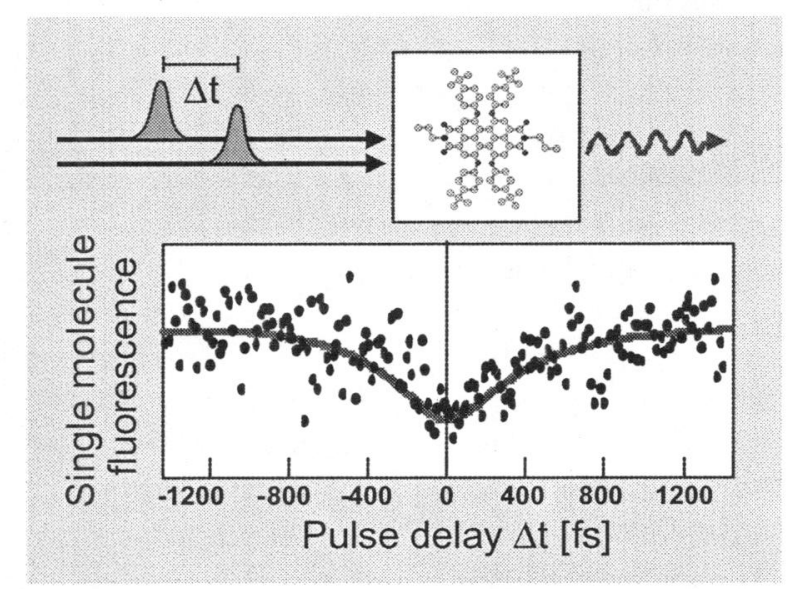

Figure.

Two intense femtoseconde pulses (blue) excite a chosen single molecule in a sample. The electronic transition is saturated. The excited state couples on fs-ps time scale to other vibrational states, which decay through spontaneous emission (ns). The spontaneously emitted photons are detected (red). The balance between stimulated and spontaneous processes in the molecule depends on the chosen pulse sequence and the molecular vibrational decay time. The experiment clearly shows how the fluorescence signal of a singlel molecule (blue dots) increases with the pulse delay. The response time (here $\sim 350 \mathrm{fs}$, red line) is characteristic for each individual molecule.

The main strength of our novel approach is towards ultrafast processes in extended mult i-chromophoric molecular assemblies. As a first step in this direction, we have studied excitonically coupled systems consisting of 2 and 3 rigidly linked perylene-diimide units in a head to tail configuration. We observe superradiance and inhibited intramolecular decay and reveal discrete jumps in femtosecond response upon break-up of the strong coupling. Recent progress in this ultrafast direction will be discussed.

[1] EM.P.H. van Dijk, J. Hernando, et al. Single-Molecule Pump -Probe Detection Resolves Ultrafast Pathways in Individual and Coupled Quantum Systems, Phys. Rev. Lett. in print. 